\title{
Leiomiomatosis Peritoneal Diseminada
}

\author{
Disseminated Peritoneal Leiomyomatosis
}

\author{
Hugo Benítez Cáceres",**; Vania Van Der Linde Rosemberg** \& Roxana Parra Lara**
}

BENÍTEZ, C. H.; VAN DER LINDE, R. V. \& PARRA, L. R. Leiomiomatosis peritoneal diseminada. Int. J. Morphol., 32(2):666-670, 2014.

RESUMEN: La leiomiomatosis peritoneal diseminada (LPD) es una entidad infrecuente que se caracteriza por la presencia de numerosas lesiones constituidas por haces de músculo liso peritoneal o subperitoneal. Estas lesiones expresan receptores hormonales, desarrollándose generalmente en pacientes en edad reproductiva. En nuestra revisión bibliográfica encontramos reportes desde el año 1976 y hasta el año 2011 aproximadamente 140 casos habían sido reportados en la literatura. Se presenta un caso de LPD diagnósticado en paciente de sexo femenino de 40 años de edad. Se describen datos quirúrgicos, examen anatomopatológico macro y microscópico con fotografías digitales, junto con una revisión de diferentes aspectos clínicos de la enfermedad.

PALABRAS CLAVE: Leiomiomatosis; Leiomioma.

\section{INTRODUCCIÓN}

La Leiomatosis peritoneal diseminada (LPD) es una entidad infrecuente que se caracteriza por la presencia de numerosos nódulos de músculo liso peritoneales o subperitoneales. Estos tumores expresan receptores hormonales, desarrollándose generalmente en pacientes en edad reproductiva, siendo su aparición independiente en muchos casos de la condición de leiomiomatosis uterina previa (Bekkers et al., 1999; Halama et al., 2005). Suele ser un hallazgo radiológico o quirúrgico, ya que la mayoría de los pacientes son asintomáticos.

Se encuentran en la bibliografía reportes desde el año 1976 (Winn et al., 1976) y hasta el año 2011 aproximadamente 140 casos habían sido reportados en la literatura (Momtahan et al., 2011). Se presenta un caso de leiomiomatosis peritoneal diseminada diagnósticado en el Laboratorio Servicios Anatomopatológicos, Antofagasta.

\section{REPORTE DE CASO}

Mujer de 40 años, con menarquia a los 12 años y períodos menstruales regulares. Sus antecedentes quirúgicos incluyeron un parto vía vaginal, dos cesáreas, la última con salpingoligaduras cinco años antes, y ooforectomía por quiste de ovario derecho, posteriormente ese mismo año. La pa- ciente no reporta alergias ni antecedentes mórbidos e indica ser fumadora. Ingresa para cirugía electiva con diagnóstico pre operatorio de miomatosis uterina. Al examen físico preoperatorio indica dolor en región suprapúbica. En el acto quirúrgico se observa siembra peritoneal en epiplón, con múltiples leiomiomas nodulares firmes, de aspecto miomatoso. Se realiza histerectomía total y omentectomía. El tiempo operatorio fue de dos horas y en el post operatorio no se reportaron complicaciones. Fue dada de alta al segundo día postoperatorio.

En el estudio anatomopatológico diferido se describe la muestra constituída por útero de 110x60x45 mm y de $139 \mathrm{~g}$, con trompa derecha adherida de $50 \mathrm{~mm}$ de longitud por $10 \mathrm{~mm}$ de diámetro transverso mayor. Al corte, el espesor del miometrio fue de 17 a $27 \mathrm{~mm}$ y se observaron varios nódulos fasciculados que midieron de 3 a $18 \mathrm{~mm}$ de diámetro mayor, de ubicación intramural, subserosa y submucosa (Fig. 1-A). Se observó endometrio atrófico y cavidad uterina conservada. En la cara anterior del útero se identificó una cicatriz de histerectomía. El cuello midió 35 $\mathrm{mm}$ de longitud y $38 \mathrm{~mm}$ de diámetro. El orificio externo se observó transversal, de $8 \mathrm{~mm}$. Separadamente se recibió un fragmento de omento que 170x90x35 mm con múltiples nódulos fasciculados que midieron entre 2x2x1,5 mm y 45x44x41 mm de diámetro mayor (Fig. 1-B). Además, se recibieron 10 nódulos fasciculados que midieron entre

\footnotetext{
* Laboratorio de Anatomía Patológica y Citopatología Servicios Anatomopatológicos Ltda., Antofagasta, Chile.

*** Unidad de Anatomía Patológica, Hospital Regional Antofagasta, Antofagasta, Chile.
} 
$15 \times 15 \times 13 \mathrm{~mm}$ y $40 \times 38 \times 35 \mathrm{~mm}$ de diámetro mayor, junto a un pequeño racimo de nódulos que midieron entre 2 y 20 $\mathrm{mm}$, con un volumen de 50x20 mm (Fig. 1-C y 1-D).

En el examen microscópico se analizaron cortes histológicos representativos de útero, cuello uterino, trompa uterina, fragmentos de epiplón y lesiones nodulares, incluidas en el epiplón y recibidas separadamente. El endometrio estaba constituido por glándulas curvas, elongadas, revestidas por epitelio columnar, en partes pseudoestratificado y estroma con edema variable. El cuello uterino se observó revestido por epitelio columnar y escamoso estratificado no queratinizado, tanto nativo como metaplásico, sin alteraciones en la zona de unión escamo-columnar, con moderada dilatación de glándulas endocervicales. Segmento de pared de trompa uterina, revestidas por epitelio columnar ciliado, dentro de límites histológicos normales. Los diagnósticos histopatológicos correspondientes a lo observado hasta aquí fueron: endometrio en fase proliferativa; ectasia glandular quística endocervical uterina; epitelio de revestimiento endocervical uterino, tanto escamoso como glandular dentro de límites histológicos normales y trompa uterina de estructura general conservada.

Al comparar todas las lesiones nodulares, tanto las uterinas, como las peritoneales, las descritas en el epiplón y las recibidas por separado, observamos a menor aumento (4x) que correspondían a lesiones medianamente hipercelulares, constituidas por fascículos de músculo liso, tejido conectivo y numerosos vasos sanguíneos de paredes finas. Con aumento intermedio (10x), se reconoció la disposición fascicular de los haces de musculo liso, tanto en las lesiones del útero, como en las peritoneales (Fig. 2-A y 2-B). Notamos que el depósito de material colagenoso hialino denso extracelular era mayor en los tumores uterinos, mientras que el edema intercelular (cambios hidrópicos) era

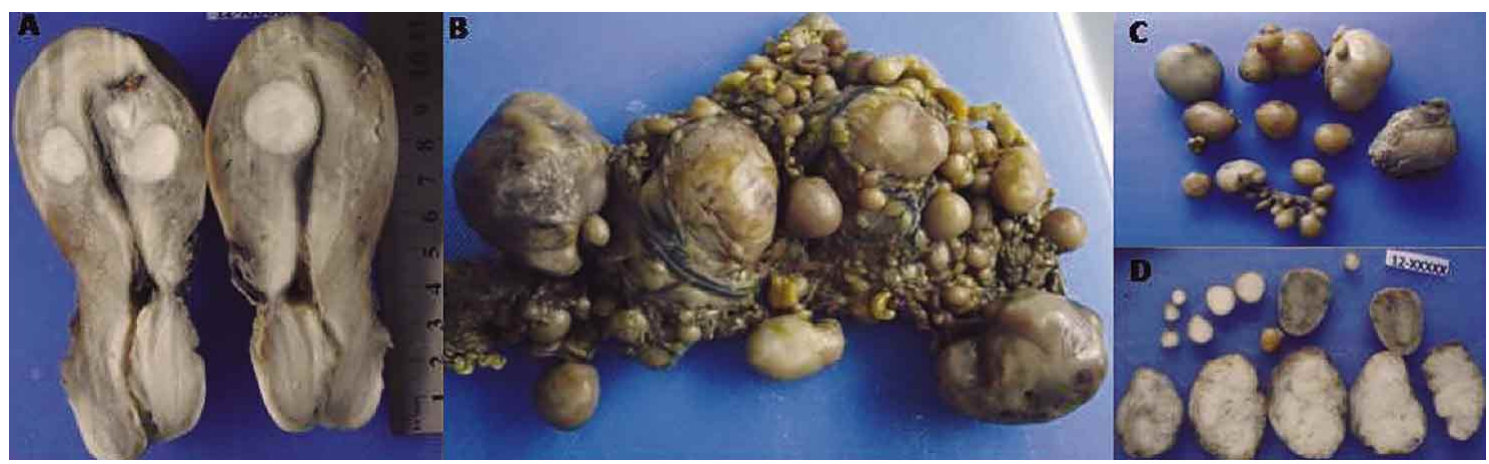

Fig. 1. A. Leiomiomas uterinos. B. Múltiples nódulos observados en superficie peritoneal. C. Nódulos individuales extirpados en la cirugía. D. Cortes seriados de nódulos.

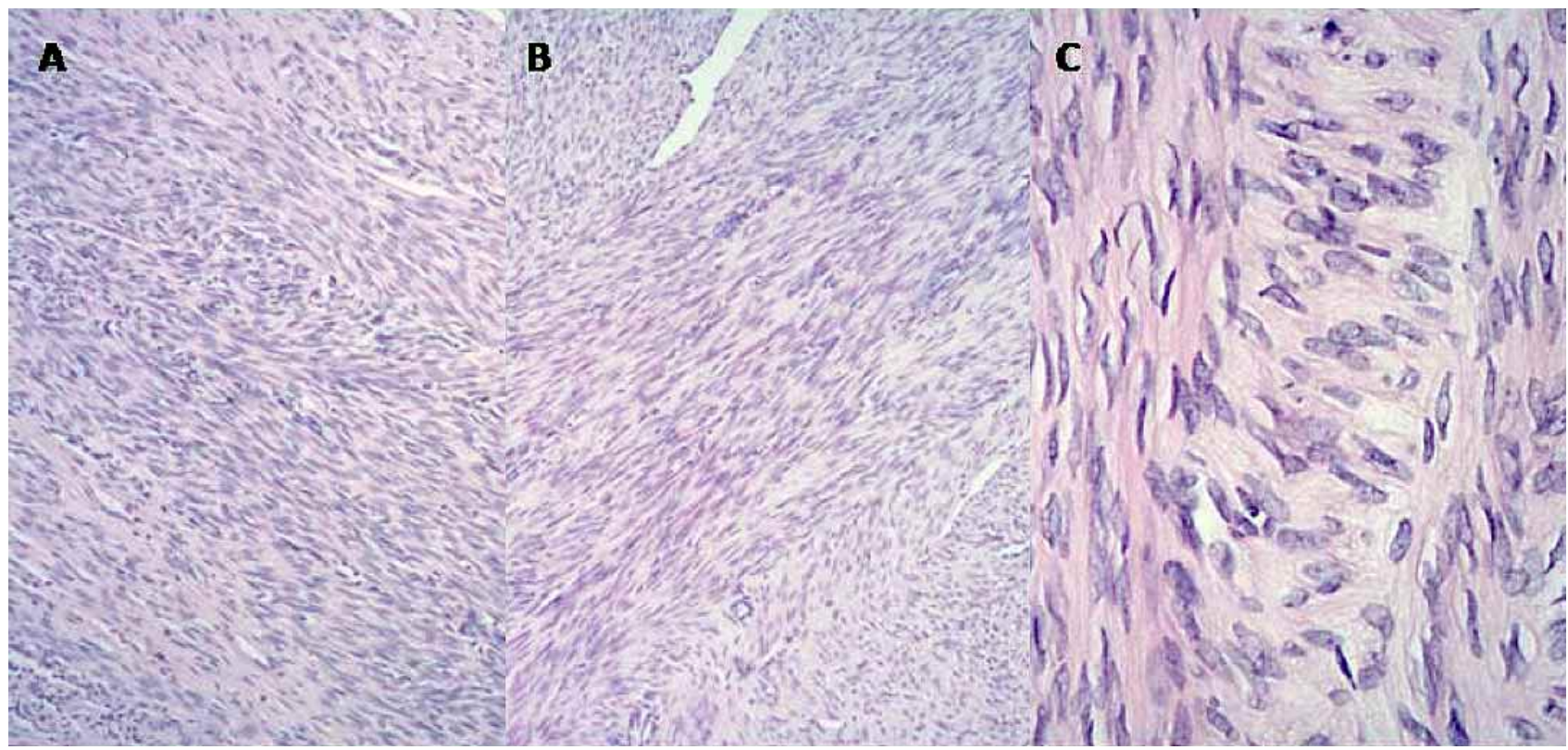

Fig. 2. Disposición fascicular en ambas localizaciones A. Leiomioma uterino (10x, H-E). B. Nódulo peritoneal (10x, H-E). C. Composición tisular similar en ambas lesiones (40x, H-E). 
más notorio en las lesiones peritoneales. A mayor aumento (40x) todas las lesiones, independientemente de su origen, estaban compuestas por células fusadas medianas, uniformes, de límites celulares poco definidos y núcleos elongados, vesiculosos, algunos con nucléolo visible y citoplasma abundante eosinófilo fibrilar (Fig. 2-C). En todos los casos, se observó leve infiltrado de predominio linfocitario, y algunos mastocitos. La inmunoexpresión observada para los recepto- res nucleares de estrógeno (clon SP1) y progesterona (clon 636) en los tumores uterinos y peritoneales fue idéntica (Figs. 3 A, B, C y D), asimismo en cuanto a la expresión de filamentos de actina (clon HHF35) (Fig. 4 A y B). En ninguna de las lesiones se observó atipias, áreas de necrosis ni mitosis significativas (menos de 2 mitosis en 10 campos de aumento mayor). Tras lo observado se concluyó: leiomiomatosis peritoneal diseminada y leiomiomatosis uterina.

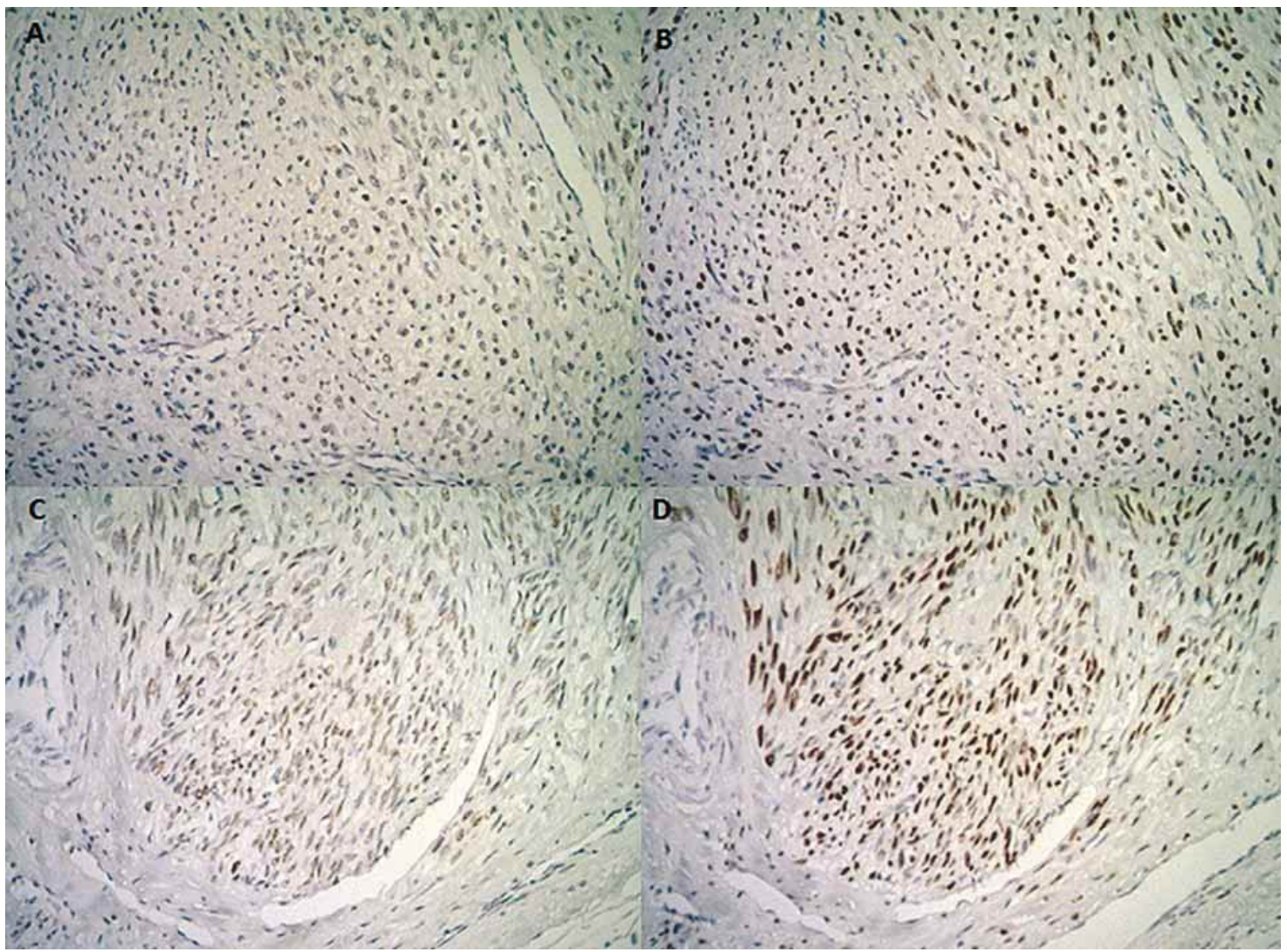

Fig. 3. Expresión de receptores hormonales, inmunohistoquímica (20x). A. Receptores de estrógeno, leiomioma uterino. B. Receptores de progesterona, leiomioma uterino. C. Receptores de estrógenos, nódulo peritoneal D. Receptores de progesterona, nódulo peritoneal.

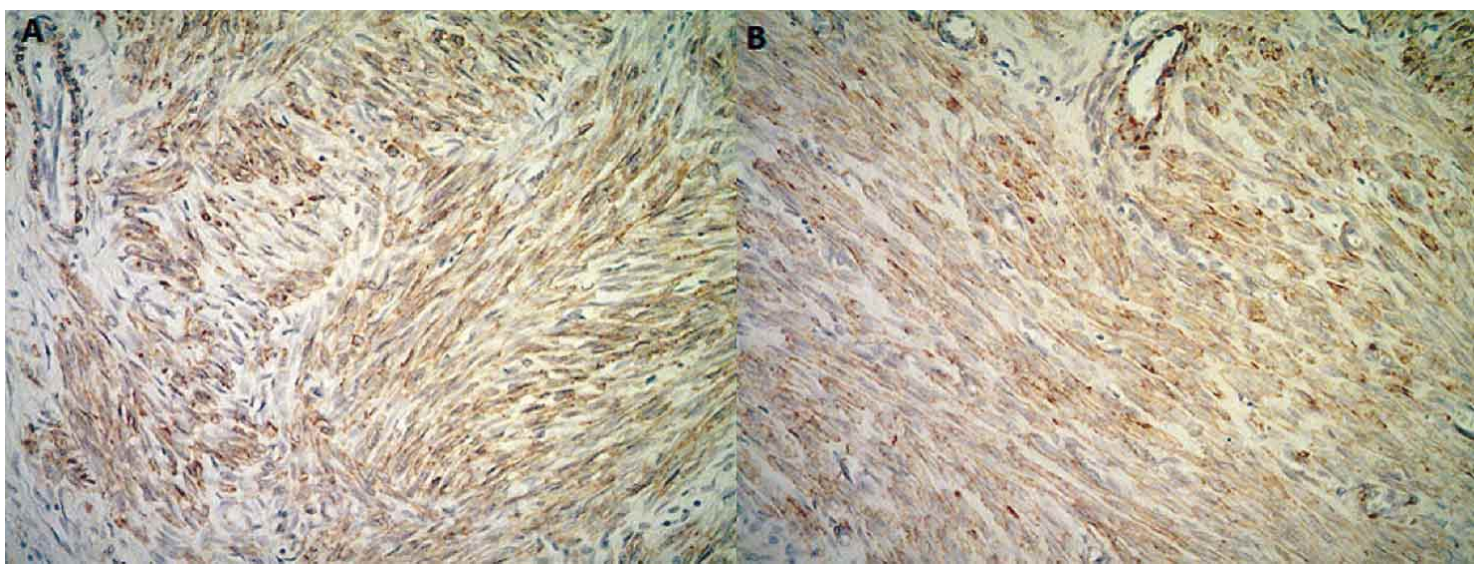

Fig. 4. Expresión de filamentos de actina musculo específica (20x). A. Leiomioma uterino. B. Nódulo peritoneal. 


\section{DISCUSIÓN}

La Leiomatosis peritoneal diseminada (LPD) es una entidad infrecuente que se caracteriza por la presencia de numerosos lesiones nodulares de músculo liso peritoneales o subperitoneales, pudiéndose desarrollar en pacientes de ambos sexos. Se define como una condición benigna, sin embargo se ha reportado en la literatura casos de progresión maligna (Alaniz Sánchez \& Castañeda Delgado, 1994; Rapagliesi et al., 1996; Rivero et al., 1998; Bekkers et al.). Estos tumores expresan receptores hormonales, desarrollándose generalmente en pacientes en edad reproductiva, siendo su aparición independiente en muchos casos de la condición de leiomiomatosis uterina previa (Bekkers et al.; Halama et al.). Suele ser un hallazgo radiológico o quirúrgico, ya que la mayoría de los pacientes son asintomático. En pacientes sintomáticos se puede observar dolor en la región supra púbica u obstrucción intestinal (Nofuentes et al., 2011). En nuestra revisión bibliográfica encontramos reportes desde el año 1976 (Winn et al.) y hasta el año 2011 aproximadamente 140 casos habían sido reportados en la literatura (Momtahan et al.).

En cuanto a una problable etiología genética, la leiomiomatosis hereditaria o Sindrome de Reed, fue descrita por Reed et al. (1973), involucrando la aparición de leiomiomas principalmente en el útero y la piel (Emer et al., 2011), habiéndose postulado un mecanismo de transmisión autosómica dominante de penetrancia variable. Se ha relacionado a esta condición con una mutación del gen que codifica la enzima fumarato hidratasa, relacionándose además con la aparición de cáncer de células renales (Albercht, 1999; Toro et al., 2003). Esta relación no ha sido comprobada específicamente en LPD, sin embargo, en el año 2005, Halama et al., reportaron un caso familiar en el que se encontraron seis miembros afectados, tres de ellos hombres, pudiendo relacionar con el diagnóstico además a tres miembros fallecidos, a través de los hallazgos de autopsia.

Además de la LPD, existen otras localizaciones inusuales para estos tumores, la liomiomatosis intravenosa (Atienza Cuevas et al., 2005) y el liomioma metastático benigno, que se encuentra en pacientes con historia de leiomioma uterino, siendo el órgano más afectado el pulmón (Roncoroni et al., 1988; Torres Gómez et al., 2007; Vieira Sabas et al., 2009).

Aunque la etiología de la LPD sigue siendo desconocida, algunos autores han sugerido como mecanismo patogénico, la metaplasia de células pluripotenciales con diferenciación miofibrosa del epitelio mülleriano, distribuido durante la embriogénesis en el mesénquima subperitoneal, y que tanto el estímulo hormonal como la predisposición genética, serían los factores determinantes en la tumorogénesis. Otros autores refutan esta teoría, ya que de ser exacta todos los nódulos en una LPD deberían ser poblaciones policlonales de células, sin embargo, se ha determinado citogenéticamente que son lesiones monoclonales con inactivación del mismo cromosoma X parental (Quade et al., 1997). En tercer lugar, se ha planteado ampliamente la etiología iatrogénica, en el caso de la fragmentación de un mioma en una cirugía laparoscópica, lo que se corresponde mejor con la naturaleza monoclonal de los tumores (Al Talib \& Tulandi, 2010; Ordulu et al., 2010).

A pesar de su localización, la LPD es histológicamente similar a los leiomiomas uterinos, que están formados de haces de musculatura lisa, desordenados y más celulares que el miometrio normal. Puede haber degeneración quística con contenido líquido seroso o calcificación. Las células muestran bordes difusos y abundante citoplasma eosinofílico y sus núcleos son elongados y de cromatina fina. El estudio inmunohistoquímico de estas lesiones corresponde a su origen en fibras musculares lisas: vimentina y actina musculo específica, y actina para músculo liso positiva. Estos tumores demuestran en la inmuhistoquímica la presencia de receptores hormonales estrogénicos y de progesterona, siendo hormonorespondedores en su gran mayoría.

El tratamiento de la LPD se establece según edad, estado hormonal y reproductivo y la sintomatología del paciente. La indicación va desde la observación y control ecográfico en pacientes asintomáticos, hasta la resección de los nódulos con salpingo-ooforectomía, histerectomía y linfadenectomía cuando las lesiones son recurrentes o aumentan demasiado de tamaño y/o hay sospecha de malignización. Se indica en algunas pacientes tratamiento hormonosupresor (García et al., 2001). Se ha reportado el uso de acetato de megestrol para lesiones residuales o se puede evaluar terapia preoperatoria con análogos de GnRH para facilitar la resección completa de los nódulos (Nofuentes et al.). Finalmente, se recomienda el seguimiento de esto pacientes por la posibilidad de recurrencia y malignización

BENÍTEZ, C. H.; VAN DER LINDE, R. V. \& PARRA, L. R. Disseminated peritoneal leiomyomatosis. Int. J. Morphol., 32(2):666-670, 2014.

SUMMARY: The disseminated peritoneal leiomyomatosis is a rare occurrence characterized by the presence of numerous 
smooth muscle nodules on peritoneal or subperitoneal surfaces. These tumors express hormone receptors and usually develop in patients of reproductive age. In our literature review we found reports from 1976 and until 2011, describing about 140 cases. We report a case diagnosed as disseminated peritoneal leiomyomatosis in a 40-year-old woman. We describe surgical data, macro and microscopic pathological examination and we present photographs and a review of different clinical aspects of the disease.

\section{KEY WORDS: Leiomyomatosis, Leiomyoma.}

\section{REFERENCIAS BIBLIOGRÁFICAS}

Alaniz Sánchez, A. \& Castañeda Delgado, Y. Leiomiomatosis peritoneal diseminada con degeneración maligna: informe de un caso. Ginecol. Obstet. Méx., 61(11):336-40, 1994.

Albretcht, S. Neoplasias and hyperplasias of neural and muscular origin. En: Freedberg, I. M.; Eisen, A. Z.; Wolff, K.; Austen, K. F.; Golsmith, L. A.; Katz, S. I. \& Fitzpatrick, T. B. (Eds.). Fitzpatrick's Dermatology in General Medicine. $5^{\text {th }}$ ed. New York, McGraw-Hill, 1999. pp.1210-2.

Al-Talib, A. \& Tulandi, T. Pathophysiology and possible iatrogenic cause of leiomyomatosis peritonealis disseminata. Gynecol. Obstet. Invest., 69(4):239-44, 2010.

Atienza Cuevas, L.; Añón Requena, M. J.; Guerrero Cauqui, R. \& Palomo González, M. J. Leiomiomatosis intravenosa uterina. Presentación de un caso con afectación paratubárica y ovárica. Rev. Esp. Patol., 38(4):238-41, 2005.

Bekkers, R. L.; Willemsen, W. N.; Schijf, C. P.; Massuger, L. F.; Bulten, J. \& Merkus, J. M. Leiomyomatosis peritonealis disseminata: does malignant transformation occur? A literature review. Ginecol. Oncol., 75(1):158-63, 1999.

Emer, J. J.; Solomon, S. \& Mercer, S. E. Reed's Syndrome: A Case of Multiple Cutaneous and Uterine Leiomyomas. J. Clin. Aesthet. Dermatol., 4(12):37-42, 2011.

García, M.; Viciano, V.; Aguiló, J.; Torro, J.; Medrano, J.; Ferri, R. J.; Garay, M.; Gómez, E. \& Ricart, M. Leiomiomatosis peritoneal diseminada. A propósito de un caso y revisión bibliográfica. Cir. Esp., 69(6):610-2, 2001.

Halama, N.; Grauling-Halama, S. A. \& Daboul, I. Familial clustering of Leiomyomatosis peritonealis disseminata: an unknown genetic syndrome? BMC Gastroenterol., 5:33, 2005.

Momtahan, M.; Nemati, M. \& Safaei, A. Disseminated peritoneal leiomyomatosis. Iran J. Med. Sci., 36(1):57-9, 2011.

Nofuentes, C.; Mella, M.; Pérez, S.; Soliveres, E.; Hernández, A. \& García, S. Leiomiomatosis peritoneal difusa. Acta Gastroenterol. Latinoam., 41(3):238-41, 2011.
Ordulu, Z.; Dal Cin, P.; Chong, W. W.; Wai Choy, K. W.; Lee, C.; Muto, M. G.; Quade, B. J. \& Morton, C. C. Disseminated peritoneal leiomyomatosis after laparoscopic supracervical hysterectomy with characteristic molecular cytogenetic findings of uterine leiomyoma. Genes Chromosomes Cancer, 49(12):1152-60, 2010.

Quade, B. J.; McLachlin, C. M.; Soto-Wright, V.; Zuckerman, J.; Mutter, G. L. \& Morton, C. C. Disseminated peritoneal leiomyomatosis. Clonality analysis by $\mathrm{X}$ chromosome inactivation and cytogenetics of a clinically benign smooth muscle proliferation. Am. J. Pathol., 150(6):2153-66, 1997.

Raspagliesi, F.; Quattrone, P.; Grosso, G.; Cobellis, L. \& Di Re, E. Malignant degeneration in leiomyomatosis peritonealis disseminata. Ginecol. Oncol., 61(2):272-4, 1996.

Reed, W. B.; Walker, R. \& Horowitz, R. Cutaneous leiomyomata with uterine leiomyomata. Acta Derm. Venereol., 53(5):40916, 1973.

Rivero, J.; Rivero, J.; Arbona, E. \& Ruiz, M. E. Leiomiomatosis peritoneal diseminada maligna. Gac. Med. Caracas, 106(4):552-4, 1998.

Roncoroni, A. J; Barcat, J. A; Berizzo, E. E. A; \& Croxatto, J. O. Leiomiomatosis pulmonar nodular leiomioma metastasiante benigno. Medicina (B. Aires), 48(2):179-85, 1988.

Toro, J. R.; Nickerson, M. L.; Wei, M. H.; Warren, M. B.; Glenn, G. M.; Turner, M. L.; Stewart, L.; Duray, P.; Tourre, O.; Sharma, N.; Choyke, P.; Stratton, P.; Merino, M.; Walther, M. M.; Linehan, W. M.; Schmidt, L. S. \& Zbar, B. Mutations in the fumarate hydratase gene cause hereditary leiomyomatosis and renal cell cancer in families in North America. Am. J. Hum. Genet., 73(1):95-106, 2003.

Torres Gómez, F. J.; Arroyo Tristán, A.; \& Torres Oliver, F. J. Leiomioma benigno metastático de localización pulmonar. Arch. Bronconeumol., 43(1):52-3, 2007.

Vieira Sabas, C.; França, J. C. Q.; Fé, J. A. M. M.; Santos, L. G. \& Almeida, N. M. G. Leiomioma uterino metastatizante benigno, relato de dois casos. Rev. Bras. Ginecol. Obstet., 31(8):4114, 2009.

Winn, K. J.; Woodruff, J. D. \& Parmley, T. H. Electronmicroscopic studies of leiomyomatosis peritonealis diseminata. Obstet. Gynecol. 48(2):225-7, 1976.

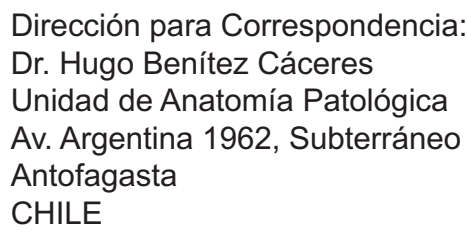

Recibido : 02-02-2013

Aceptado: 02-05-2014

Email: chanitaparra@yahoo.com 